15

\title{
Высокочувствительная экспрессная нелинейно-оптическая диагностика кристаллического состояния гетероструктур типа сфралерита
}

\author{
(C) М.Ф. Ступак, ${ }^{1,3}$ Н.Н. Михайлов, ${ }^{2,3}$ C.А. Дворецкий, ${ }^{2,4}$ С.Н. Макаров, ${ }^{1}$ А.Г. Елесин, ${ }^{1}$ А.Г. Верхогляд ${ }^{1}$ \\ ${ }^{1}$ Конструкторско-технологический институт научного приборостроения СО РАН, \\ 630058 Новосибирск, Россия \\ ${ }^{2}$ Институт фризики полупроводников СО РАН, \\ 630090 Новосибирск, Россия \\ ${ }^{3}$ Новосибирский государственный университет, \\ 630090 Новосибирск, Россия \\ ${ }^{4}$ Томский государственный университет, \\ 634050 Томск, Россия \\ e-mail: stupak@tdisie.nsc.ru
}

Поступило в Редакцию 13 февраля 2021 г.

В окончательной редакции 16 мая 2021 r.

Принято к публикации 11 июня 2021 г.

Представлены характеристики высокочувствительного экспрессного стенда нелинейно-оптической диагностики кристаллических структур типа сфалерита с помощью генерации второй гармоники. Проведен анализ возможностей количественной и качественной характеризации особенностей кристаллических параметров слоев гетероэпитаксиальных структур $\mathrm{Cd}_{x} \mathrm{Hg}_{1-x} \mathrm{Te}$ на подложках из $\mathrm{GaAs}$ с ориентацией (013). Получены результаты по отклонениям ориентации в слоях от ориентации подложки, возникшим при эпитаксии, по определению существования напряжений. Высокая чувствительность стенда позволила выявить наличие/отсутствие микроучастков с разупорядоченной кристаллической структурой. Приведены экспериментальные результаты обратимой модификации in situ кристаллического состояния структур $\mathrm{Cd}_{x} \mathrm{Hg}_{1-x} \mathrm{Te}$ при кратковременном локальном лучевом воздействии лазерного излучения повышенной мощности. Приведены новые экспериментальные данные, показывающие, что компоненты тензора нелинейной восприимчивости $\chi_{x y z}(\omega)$ кристаллической гетероструктуры $\mathrm{Cd}_{x} \mathrm{Hg}_{1-x}$ Te зависят от состава и на порядок превосходят по величине аналогичные компоненты тензора в CdTе и GaAs.

Ключевые слова: кристаллы класса сфалерита, вторая гармоника, азимутальные угловые зависимости, тензор нелинейной восприимчивости, напряжения, микроучастки, лучевой нагрев, гетероструктуры $\mathrm{Cd}_{x} \mathrm{Hg}_{1-x} \mathrm{Te}$.

DOI: $10.21883 /$ JTF.2021.11.51546.34-21

\section{Введение}

Для определения структурного совершенства кристаллов и их дефектности используются хорошо зарекомендовавшие себя методы, основанные на дифракции рентгеновских лучей, электронных и нейтронных пучков. Эти методы имеют высокую чувствительность. Однако для их реализации необходимо специальное оборудование, создание защиты персонала, а также существует специфика в подготовке образцов для проведения исследований. Отдельная проблема для этих методов создание малых зон контроля вещества. Например, глубина проникновения рентгеновского излучения может составлять сотни микрон.

Более трех десятков лет назад был предложен и исследован эффективный метод диагностики кристаллического совершенства нецентросимметричных кристаллов - метод генерации второй гармоники (ВГ) [1]. Генерация ВГ как метод контроля кристаллической структуры оказывается более быстрым и удобным методом для проведения исследований с целью получения экспрессной информации, чем традиционные электронные, рентгеновские и другие дифракционные методы. Зависимость интенсивности поляризационных компонент ВГ от взаимной ориентации поляризации возбуждающего излучения относительно кристаллофизических осей, когда регистрируется поляризация ВГ, параллельная или перпендикулярная азимутально меняющейся линейной поляризации возбуждающего излучения (в тексте далее - угловая или азимутальная зависимость), позволяет при сравнении экспериментальных и модельных данных в заданной локальной области получать количественную информацию о кристаллическом состоянии приповерхностного слоя, его ориентации и ряд других характеристик с погрешностью не более градуса [1-11]. Использование диагностических возможностей метода ВГ находит широкое применение, например, при исследова- 
нии электронных и магнитных свойств материалов по характеристикам обнаруженной индуцированной магнитным полем генерации второй оптической гармоники в диамагнитных и парамагнитных полупроводниках $[12,13]$.

Отметим, что метод ВГ позволяет при перемещении сфокусированного лазерного луча по двум координатам поверхности образца получать данные с большой площади с высоким локальным разрешением в приповерхностном слое кристалла [5,14].

Повышение чувствительности метода ВГ позволяет выявить особенности тонкой структуры кристаллического совершенства кристаллов, особенно для многокомпонентных и многослойных структур. К такому типу структур относятся гетероэпитаксиальные слои (ГЭС) теллурида кадмия и ртути (КРТ, HgCdTe), выращиваемые методом молекулярно-лучевой эпитаксии (МЛЭ) на подложках из $\mathrm{GaAs}$ и $\mathrm{Si}$, имеющие класс симметрии сфалерита или цинковой обманки. Получение высокого качества этих структур для последующих практических применений в ИК детекторах [15] требует решения проблем, связанных с большим рассогласованием параметров решеток и неизовалентностью сопрягаемых материалов соединений КРТ и подложек из GaAs и $\mathrm{Si}$ [16]. Проведенные различными методами исследования выявили образование большого количества дефектов кристаллической структуры и выявили связь их образования с условиями роста [17-21]. Проведенные ранее исследования с помощью генерации второй гармоники зондирующего излучения позволили определить ориентацию и кристаллическое состояние слоев в ГЭС КРТ МЛЭ на подложках из (013) GaAs, показали высокую эффективность метода ВГ при экспериментальной отработке режимов создания высококачественных гетероструктур [9-11]. Полученные данные позволяют прогнозировать применение метода ВГ для исследования качества последовательных стадий процессов роста слоев ГЭС КРТ МЛЭ для определения оптимальных условий проведения процессов и требований к подложке. Однако для расширения возможностей исследований кристаллической структуры, ее дефектности и наблюдения более тонких эффектов требуется повышение чувствительности нелинейно-оптической диагностики.

Целью настоящей работы является представление характеристик высокочувствительного экспрессного стенда для проведения нелинейно-оптической диагностики (НОД) и изложение результатов его применения для более информативной характеризации кристаллического качества ГЭС КРТ МЛЭ и подложечного материала этих структур по генерации ВГ на отражение, получение новой информации о наличии/отсутствии напряжений в структурах и подложке, выявление разориентированных микроучастков, обратимое лучевое воздействие и оценки отношений тензора $\chi_{x y z}(\omega)$ в слоях КРТ и подложке GaAs.

\section{1. Особенности возбуждения и регистрации сигналов ВГ в кристаллах GaAs, CdTe, KPT}

Расчет интенсивности сигнала ВГ, определяемой компонентами тензора нелинейной восприимчивости $\chi_{x y z}(\omega)$ в кристаллах класса сфалерита (международная классификация - класс $\overline{4} 3 m)$, подробно описан в $[1,2]$. Для сравнения расчетных данных и экспериментальных результатов поведения азимутальных зависимостей сигнала ВГ в конкретной геометрии и для конкретных срезов кристалла применяется численное моделирование $[3,5,11]$.

Для выращивания гетероструктур КРТ методом МЛЭ нами используются подложки из GaAs c ориентацией (013). Для сохранения ориентации и решения проблемы большого несоответствия параметров решетки GaAs и КРТ в 13.6\% последовательно выращивают буферные слои ZnTe и CdTe. Таким образом, создается „альтернативная“ подложка CdTe/ZnTe/GaAs, которая сохраняет ориентацию (013) и обеспечивает существенно меньшее несоответствие параметров решетки с КРТ (менее $0.3 \%$ ).

Для составляющих компонентов гетероструктур КРТ, таких как подложка $\mathrm{GaAs}$, слои CdTe и $\mathrm{ZnTe}$, поглощением в области возбуждающего излучения $\sim 1 \mu \mathrm{m}$ можно пренебречь и учитывать только потери на френелевских отражениях. При анализе справочных и литературных данных оптических характеристик полупроводниковых материалов отмечается значительный разброс измеренных значений коэффициентов отражения, диэлектрической проницаемости, коэффициентов поглощения, что объясняется различными технологиями получения исследуемого материала, различными условиями постановки и проведения экспериментов, а также разными технологическими приемами обработки поверхности образцов. Особенно важно состояние поверхности образцов в области фундаментального поглощения, когда взаимодействие света с веществом происходит в весьма тонком приповерхностном слое [22]. В области фундаментального поглощения на длине волны второй гармоники $(\lambda=0.53 \mu \mathrm{m})$ глубина проникновения по справочным и нашим данным составляет для СdTe $\sim 130 \mathrm{~nm}$, для $\mathrm{GaAs}-\sim 140 \mathrm{~nm}$, для $\mathrm{ZnTe}-\sim 210 \mathrm{~nm}$. Эти цифры находятся в удовлетворительном соответствии со справочными и литературными данными [22-24]. Слои КРТ непрозрачны и для возбуждающего излучения на $\lambda=1.064 \mu \mathrm{m}$, и для его второй гармоники. Для видимого диапазона глубина проникновения существенно зависит от состава верхнего слоя КРТ $[23,24]$. Замеренная нами глубина проникновения для верхнего варизонного слоя наших гетероструктур по амплитуде в области $1 \mu \mathrm{m}$ не превышает $0.2 \mu \mathrm{m}$, а длина поглощения ВГ по амплитуде $\leq 0.03 \mu \mathrm{m}$, что хорошо согласуется с данными $[23,24]$. Это означает, что в наших экспериментах сигналы ВГ от гетероструктуры КРТ и от 
подложки GaAs этой гетероструктуры являются чисто отраженными.

Учитывая интерференционную природу отраженной второй гармоники, ее эффективная глубина генерации определяется в случае прозрачной среды ее когерентной длиной $d \approx \lambda / 4 \pi n(\omega)$, при сильном поглощении глубина генерации $d$ определяется минимальным значением величин $\alpha^{-1}(\omega)$ и $\alpha^{-1}(2 \omega)$ [1], где $\lambda$ - длина волны основного излучения, $n-$ показатель преломления среды на этой длине волны, $\alpha-$ соответствующие коэффициенты поглощения на основной и удвоенной частотах. В промежуточных случаях слабого поглощения для определения эффективной глубины генерации необходимо использовать точную формулу $d=\lambda / 4(n(\omega)+n(2 \omega))[25,26]$. Проведенные оценки показали, что глубина диагностики исследуемых в сообщении образцов $\mathrm{HgCdTe}$ определяется величиной $\alpha^{-1}(2 \omega)$, глубина генерации ВГ, отраженной от подложек GaAs и слоев CdTe, определяется когерентной длиной и составляет величину $\sim 25-20 \mathrm{~nm}$.

Проведенный нами в [11] анализ поведения графиков модельных азимутальных зависимостей сигнала ВГ (поляризация ВГ параллельна или перпендикулярна азимутально меняющейся линейной поляризации возбуждающего излучения) с вариацией по углам $\Theta$ и $\varphi$ вблизи ориентации (013) показал, что эти зависимости наиболее чувствительны к небольшим отклонениям от угла $\varphi$ при нормальном падении луча. Поэтому для достижения высокой чувствительности метода ВГ при определении ориентации исследуемого приповерхностного слоя на лабораторном стенде нелинейно-оптической диагностики осуществляется нормальное падение лазерного излучения частоты w на исследуемый образец с одновременным вращением плоскости поляризации (азимутальный угол) возбуждающего излучения в диапазоне от 0 до $359^{\circ}$ и регистрацией интенсивности сигнала отраженной ВГ с поляризацией, параллельной или перпендикулярной поляризации лазерного излучения. Хотя вся необходимая информация на графиках азимутальной зависимости сигнала ВГ содержится в диапазоне 0-179 , а далее график просто повторяется, экспериментаторы используют диапазон от 0 до $359^{\circ}$ именно для контроля этой повторяемости из-за возможных апертурных погрешностей экспериментальной установки при юстировке как оптического тракта прохождения сигнала ВГ к приемной аппаратуре, так и тракта лазерного возбуждающего излучения, проходящего через вращающуюся пластинку $\lambda / 2$.

Проведенный фурье-анализ модельных азимутальных зависимостей вблизи среза (013) гетероструктуры типа сфалерита представлен на рис. 1 и демонстрирует наличие только первой, второй и третьей гармоник со своими амплитудами и фазами (см. [1]). При моделировании отсчет углов $\Theta$ и $\varphi$ проводился от базового среза (100). Амплитуда фурье-гармоник представлена в относительных единицах, поляризация отраженной ВГ параллельна поляризации возбуждающего лазерного излучения.

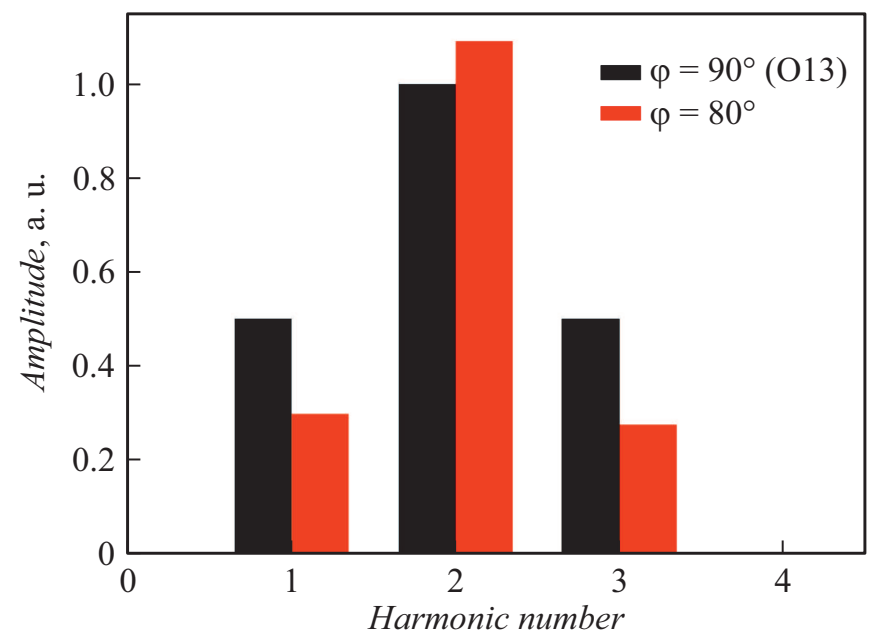

Рис. 1. Фурье-анализ модельных азимутальных зависимостей интенсивности ВГ для нормального падения луча при вариации среза вблизи (013) по углу $\varphi$.

Следует подчеркнуть, что график азимутальной зависимости сигнала ВГ для идеально точной ориентации (013) при угле $\varphi=90^{\circ}$ должен демонстрировать одинаковые амплитуды всех четырех максимумов. Было показано, что изменения сигнала ВГ по углу $\Theta$ (при фиксированном угле $\varphi=90^{\circ}$ ) вблизи среза $(013)$ приводят к практически одинаковому изменению амплитуды графиков зависимости интенсивности ВГ от азимутального угла без изменения соотношений между максимумами [11].

При диагностике полупроводниковых материалов и структур, прозрачных для основного излучения, величина регистрируемого сигнала отраженной ВГ при заданном уровне возбуждающего лазерного излучения зависит от характеристик кристалла, совершенства его приповерхностного слоя и от объемных напряжений (наведенное двулучепреломление), отражения возбуждающего излучения от тыльной поверхности образца в точке контроля $[3,5,11]$. При исследовании подложек GaAs и буферных слоев CdTe, учитывая сильное поглощение на частоте второй гармоники в прозрачных для основного излучения этих полупроводниковых материалах, можно утверждать, что сигнал ВГ соответствует точечной группе симметрии кристалла приповерхностного слоя и может, таким образом, использоваться для характеризации структурного качества слоя толщиной $0.3-1 \mu \mathrm{m}$. Величина сигнала ВГ в этом случае может более чем на порядок превосходить величину отраженного только от передней поверхности сигнала ВГ $[5,11]$. Так как слои КРТ непрозрачны для возбуждающего излучения $(\lambda=1.064 \mu \mathrm{m})$ и для его второй гармоники, имеется уникальная возможность фиксировать только отраженную ВГ как от поверхностного слоя КРТ в гетероструктуре, так и от обратной стороны подложки. Подложки GaAs имели двухстороннюю полировку. Это позволяет производить сравнительный анализ нелинейных 
восприимчивостей данных материалов, диагностировать сравнительное присутствие напряжений в подложке и в слоях гетероструктуры КРТ.

Таким образом, критерием оценки качества кристаллического состояния в данной локальной области поверхности исследуемого образца в результате анализа измеренных азимутальных зависимостей является:

а) выявление соответствия определенной точечной группе симметрии и определение с требуемой точностью абсолютной ориентации локальной кристаллической области относительно поверхности образца;

б) выявление присутствия напряжений в данной области;

в) качественная оценка уровня дефектов по сравнительному анализу амплитуд сигнала ВГ (вдоль поверхности или для однотипных образцов).

\section{2. Высокочувствительный лабораторный стенд нелинейно-оптической диагностики}

Подробное описание гетероструктуры и работы лабораторного стенда НОД, состоящего из лазерного источника, оптико-механического блока, блоков питания, блока приема и обработки информации на основе управляющего компьютера, приведено в [11]. В качестве излучателя используется импульсно-периодический YAG: Nd-лазер с диодной накачкой DUETTO-OEM V3.4 с длиной волны $1.064 \mu \mathrm{m}$, частотой повторения цуга из $\sim 10$ импульсов $50 \mathrm{kHz}$ при длительности одного импульса в цуге $\sim 10 \mathrm{ps}$. Излучение лазера фокусируется длиннофокусной линзой на поверхность тестируемого образца в пятно диаметром $200 \mu \mathrm{m}$ в соответствии с требующимся пространственным разрешением. Пластинка $\lambda / 2$ вращается по заданной программе вокруг оси лазерного луча шаговым двигателем и вращает плоскость поляризации падающего нормально на образец излучения. Специализированное параметрическое зеркало (при угле падения $45^{\circ}$ пропускает лазерное излучение и отражает его вторую гармонику) отделяет в отраженном от образца излучении сигнал ВГ и далее через анализатор, ориентация которого синхронизована с ориентацией пластинки $\lambda / 2$, и светофильтр, пропускающий только излучение ВГ, направляет по световоду сигнал ВГ на ФЭУ-130.

Нами проведена модернизация стенда, который претерпел целевые изменения, что позволило более чем на порядок снизить имевшиеся шумы и повысить уровень полезного сигнала ВГ и тем самым повысить чувствительность. Это было достигнуто в основном за счет частотной селекции и усиления полезного сигнала после фотоумножителя.

Сигнал с ФЭУ поступал теперь на селективный усилитель-нановольтметр UNIPAN-233, настроенный на частоту работы импульсно-периодического YAG:Ndлазера, равную $50 \mathrm{kHz}$. Далее отселектированный по частоте и усиленный сигнал поступал на модуль АЦП и непрерывно считывался специализированным программным обеспечением, установленным в персональном компьютере.

Повышение чувствительности стенда позволило снизить среднюю мощность зондирующего лазерного излучения. В представленных ниже экспериментальных результатах средняя мощность варьировалась от 10 до $70 \mathrm{~mW}$. Стандартное время получения экспериментального графика угловой зависимости сигнала ВГ составляло от 10 до $30 \mathrm{~s}$. Повышение почти на два порядка чувствительности стенда НОД привело к регистрации доли процента пропускаемого параметрическим зеркалом сигнала ВГ, рождаемого в кварцевой пластине $\lambda / 2$, поэтому в приемный тракт стенда после кварцевой пластины $\lambda / 2$ был поставлен обрезающий светофильтр КС-17.

Приемной аппаратурой стенда регистрировался сигнал интенсивности отраженной ВГ с поляризацией, параллельной поляризации лазерного излучения, все представленные в сообщении экспериментальные результаты соответствуют этой конфигурации.

Конструктивно оптико-механический блок стенда НОД помещен на оптической плите лазерного источника в небольшой $(50 \times 50 \times 25 \mathrm{~cm})$ светонепроницаемый кожух с входным и выходным отверстием для рабочего излучения, к выходному отверстию пристыкован легко сменяемый кожух блока крепления и юстировки исследуемого образца. Это позволило организовать штатную работу комплекса НОД в лабораторных условиях при нормальном внешнем освещении, причем операция замены и юстировки исследуемого образца занимала менее пяти минут.

Стандартное отклонение сигнала шума (с нулевым средним) соответствовало примерно 16 единицам кода АЦП, уровень характерной амплитуды сигнала ВГ на графиках находился в пределах 2000-4500 единиц кода АЦП.

Погрешность сравнения экспериментальных и модельных угловых зависимостей сигнала ВГ от образцов по углу $\varphi$ составляла один градус. Основным количественным параметром используемого метода НОД является определение с требуемой точностью абсолютной ориентации локальной кристаллической области относительно поверхности образца. В нашем случае точность определяется „частотой“ сетки сравнения по углам $\Theta$ и $\varphi$ модельных азимутальных зависимостей с экспериментальным графиком. Программно шаг сетки выбирался равным $1^{\circ}$ по углу $\varphi$. При необходимости в случае слабых напряжений шаг сетки сравнения по углам $\Theta$ и $\varphi$ модельных азимутальных зависимостей можно выбирать равным $0.1^{\circ}$, тогда точность определения абсолютной ориентации возрастает на порядок. Сравнительный анализ амплитуд экспериментального сигнала ВГ (вдоль поверхности или для однотипных образцов) может быть проведен с погрешностью менее 0.05. Таким образом, изменение количества нелинейных диполей, связанное 
с изменением плотности различных дефектов, можно фиксировать с погрешностью 0.05. С такой же погрешностью выявляется наличие/отсутствие напряжений по поднятию некоторых минимумов экспериментального графика азимутальной зависимости.

\section{3. Характеризация кристаллического состояния слоев и структур КРТ, подложек GaAs c помощью стенда НОД}

\section{1. Оценка отношений тензора $\chi_{x y z}(\omega)$ в структурах KPT и GaAs. Учет фактора переотраженных волн}

Были проведены исследования слоев и подложки гетероструктур $\mathrm{Cd}_{x} \mathrm{Hg}_{1-x} \mathrm{Te}(\mathrm{KPT}) / \mathrm{CdTe} / \mathrm{ZnTe} / \mathrm{GaAs}$, выращенных методом молекулярно-лучевой эпитаксии на установке „Обь-М“. На полуизолирующих подложках (013)GaAs последовательно выращивались буферные слои $\mathrm{ZnTe}$ и $\mathrm{CdTe}$ толщинами $30 \mathrm{~nm}$ и $5.5 \mu \mathrm{m}$ соответственно. Слои $\mathrm{Cd}_{x} \mathrm{Hg}_{1-x} \mathrm{Te}$ состояли из поглощающего рабочего слоя состава $X_{\mathrm{CdTe}}=0.22$ с толщиной $\sim 6 \mu \mathrm{m}$ и широкозонными варизонными слоями на границах рабочего слоя, в которых состав плавно изменялся от $X=0.45$ до 0.22 на начальной стадии роста КРТ на толщине $\sim 1.5 \mu \mathrm{m}$ (внутренний варизонный слой) и при его окончании от $X=0.22$ до 0.45 на толщине $\sim 0.5 \mu \mathrm{m}$ (внешний варизонный слой).

Нами была зарегистрирована существенно более высокая амплитуда сигнала ВГ от внешнего варизонного слоя $\mathrm{Cd}_{0.45} \mathrm{Hg}_{0.55} \mathrm{Te}$ поверхности гетероструктуры $\mathrm{Cd}_{x} \mathrm{Hg}_{1-x} \mathrm{Te}(\mathrm{KPT}) / \mathrm{CdTe} / \mathrm{ZnTe} / \mathrm{GaAs}$ по сравнению с нижележащими буферными слоями CdTe и подложки из GaAs [11]. Полученные результаты свидетельствуют о том, что в данном случае компоненты тензора нелинейной восприимчивости $\chi_{x y z}(\omega)$ кристаллической гетероструктуры существенно превосходят по величине аналогичные компоненты тензора в CdTe и GaAs. При измерениях на высокочувствительном стенде НОД при последующих экспериментах при фиксированном уровне чувствительности регистрирующей аппаратуры (пита-

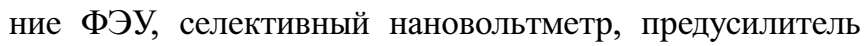
АЦП) и одинаковом уровне возбуждающего ИК излучения (средняя мощность излучения на $\lambda=1.064 \mu \mathrm{m}$ равнялась $0.06 \mathrm{~W}$ ) амплитуда сигнала отраженной от верхнего варизонного слоя КРТ второй гармоники была также значительно больше по сравнению с аналогичными сигналами ВГ от слоев CdTe, ZnTe или от обратной стороны подложки GaAs. Нами были проведены такие же измерения после удаления внешнего варизонного слоя с помощью химического травления до состава рабочего слоя. Результат диагностики представлен на рис. 2, на котором приведены в одинаковом масштабе экспериментальные графики азимутальной угловой зависимости

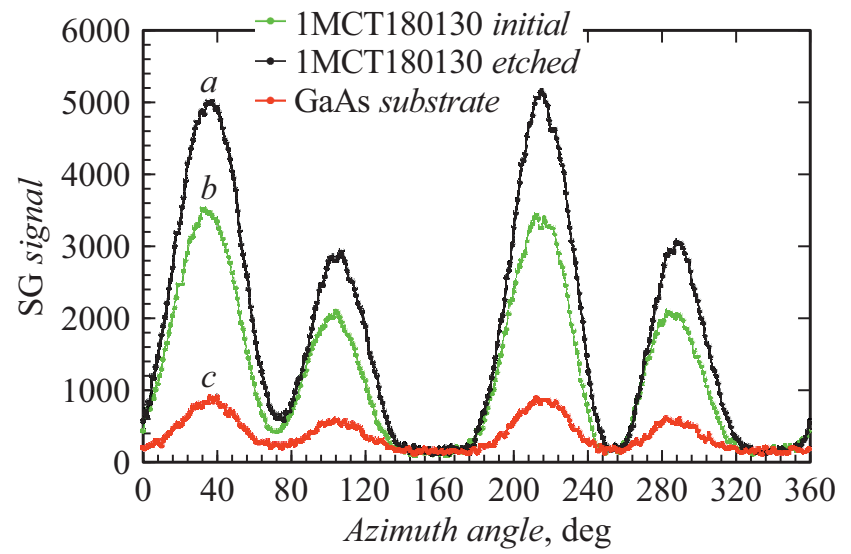

Рис. 2. Экспериментальные графики азимутальной зависимости амплитуды сигнала ВГ гетероструктуры КРТ180130: $a$ - от внешнего варизонного слоя; $b-$ после удаления внешнего варизонного слоя; $c-$ от обратной стороны подложки из GaAs. Средняя мощность излучения на $\lambda=1.064 \mu \mathrm{m}$ составила $0.06 \mathrm{~W}$. Ориентация слоев $a$ и $b$ отлична от (013) по углу $\varphi$ на $-5^{\circ}$, ориентация подложки отлична от $(013)$ по углу $\varphi$ на $-3^{\circ}$. Поляризации ВГ и лазерного излучения параллельны.

сигнала ВГ от внешнего варизонного слоя в гетероструктуре КРТ180130 $(a)$, после его удаления с помощью химического травления $(b)$ и от подложки GaAs этой гетероструктуры $(c)$. Отметим, что здесь и далее, где это необходимо, выявленные количественные характеристики ориентации исследуемых объектов приведены в подписях к соответствующим рисункам.

Из рисунка видно, что величина сигнала ВГ, отраженной от поверхности внешнего варизонного широкозонного слоя КРТ с большим содержанием кадмия, в 1.5 раза меньше соответствующего сигала ВГ, отраженного от поверхности рабочего слоя. Эти сигналы ВГ от структур КРТ более чем на порядок превосходят отраженный сигнал ВГ от тыльной стороны образца, т. е. от подложки GaAs.

На основании этих и других полученных данных, а также с учетом экспериментально зафиксированной непрозрачности слоя КРТ для возбуждающего излучения на $\lambda=1.064 \mu \mathrm{m}$ (напомним, глубина проникновения по амплитуде не превышает $0.2 \mu \mathrm{m}$, а длина поглощения ВГ по амплитуде $\leq 0.03 \mu \mathrm{m})$, можно утверждать, что сигналы ВГ от слоев гетероструктуры КРТ и от подложки GaAs являются чисто отраженными без вклада переотраженного лазерного излучения от тыльной поверхности подложки образца. Таким образом, наблюдаемое отличие амплитуды сигналов ВГ на рис. 2 и учет характерной длины поглощения по амплитуде второй гармоники в используемых подложках арсенида галлия $\approx 0.15 \mu$ m свидетельствует о том, что компоненты тензора нелинейной восприимчивости $\chi_{x y z}(\omega)$ на длине волны $\sim 1 \mu$ т кристаллической гетероструктуры КРТ более чем на порядок превосходят по величине аналогичные 
компоненты тензора кристаллической гетероструктуры $\mathrm{CdTe}$ и $\mathrm{GaAs}$, представленные в [1].

\section{2. Выявление эффекта присутствия разориентированных микроучастков в слоях КРТ}

При диагностике приповерхностных слоев отдельных исследуемых гетероструктур КРТ и при послойном химическом травлении наблюдалось увеличение шума в экспериментальных графиках азимутальной зависимости сигнала ВГ, превышающего уровень шумов всего приборного тракта. Особенно четко это увеличение было видно в области минимумов сигнала ВГ (своего рода ноль-метод). Не ставя в настоящей работе задачу определения приоритета первых работ с использованием ноль-метода при исследовании сигналов ВГ, отметим, что различные модификации такой методики для диагностики любых типов физических неоднородностей на основе „запрещенных“ сигналов ВГ широко применяются, начиная с 80-х годов прошлого века и по настоящее время (см., например, $[27,28])$. Такое увеличение шума можно связать с наличием в области измерений (диаметр падающего излучения $\sim 200 \mu \mathrm{m}$ ) присутствия разориентированных микроучастков, которые при азимутальной ориентации поляризации основного излучения и второй гармоники вызывают генерацию отличного от нуля сигнала ВГ, особенно в области минимума, в которой идеальный монокристалл не генерирует данную поляризацию ВГ. Для большинства измеряемых гетероструктур КРТ шумы азимутальной зависимости сигнала ГВ лимитировались шумами измерительного тракта. Таким образом, наблюдаемые эффекты мы называем наличием/отсутствием разориентированных микроучастков. На рис. 3 приведены в одинаковом масштабе первичные экспериментальные графики азимутальной

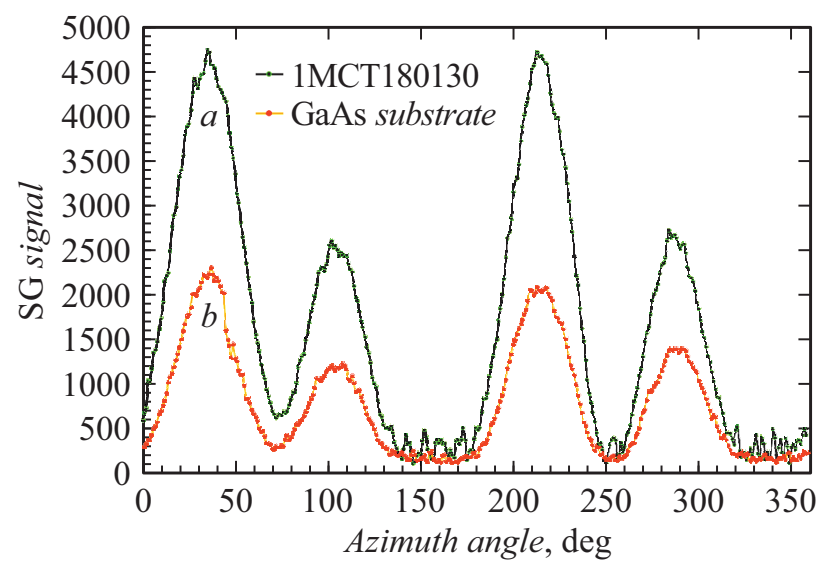

Рис. 3. Экспериментальные графики азимутальной зависимости сигнала ВГ от гетероструктуры КРТ180130: $a$ - после химического травления на глубину $\sim 5 \mu \mathrm{m} ; b-$ от подложки GaAs. Средняя мощность излучения на $\lambda=1.064 \mu \mathrm{m}$ равна $0.06 \mathrm{~W}$. Поляризации ВГ и лазерного излучения параллельны. зависимости сигнала ВГ от поверхности слоя КРТ образца КРТ180130 после химического травления на глубину $\sim 5 \mu \mathrm{m}(a)$ и от подложки GaAs этого образца $(b)$.

\section{3. Диагностика напряжений в слоях КРТ и подложечном материале}

Наблюдение напряжений в гетероструктурах и подложках осуществлялось по наличию на экспериментальных графиках ярко выраженной асимметрии минимумов угловой зависимости и изменения (поднятия) их уровней $[5,11]$. Проведенные измерения на высокочувствительном стенде НОД выявили такую же картину изменений угловой зависимости сигналов ВГ.

Проведем оценку влияния деформаций в полупроводниковых образцах на поляризационные характеристики прошедшего образец лазерного излучения на основе предложенной модели слабых напряжений [14].

Эффект слабых напряжений связан с наличием деформаций, внесенных в образец при росте или обработке. Появляющиеся в кристалле механические напряжения малы и не могут оказать влияния на класс симметрии или ориентацию кристаллографических осей в образце, но они влияют на поляризационные свойства распространяющегося в кристалле лазерного излучения. Падающая линейно поляризованная волна внутри кристалла становится эллиптически поляризованной. Среда является одноосным кристаллом с мало отличающимися показателями преломления вдоль оптической оси и в направлениях, перпендикулярных ей. Эллиптичность волны после взаимодействия с кристаллом определяется разностью показателей преломления и зависит от величины напряжений в данной точке, ориентация эллипса в пространстве определяется распределением механических напряжений в образце.

Пусть в образце, прозрачном для падающего вдоль оси $Z$ нормально на пластину излучения, возникает слабая поперечная деформация с показателем преломления вдоль нее $n_{e}$, а в поперечном направлении $n_{o}$, направленная под углом $\beta$ к оси лабораторной системы координат $Y$. Вектор Джонса падающего излучения с азимутальным углом поляризации $\alpha$ есть

$$
\left(\begin{array}{l}
V_{x} \\
V_{y}
\end{array}\right)=E_{o}\left(\begin{array}{c}
\cos \alpha \\
\sin \alpha
\end{array}\right) .
$$

Проведем расчет матрицы Джонса для образца. В образце распространяются обыкновенная и необыкновенная волны. $R(\beta)$ - матрица перехода из лабораторной системы координат в систему, связанную с векторами поляризации этих волн.

$$
R(\beta)=\left(\begin{array}{rr}
\cos \beta & \sin \beta \\
-\sin \beta & \cos \beta
\end{array}\right),
$$

следовательно, в системе координат, связанной с векторами поляризации обыкновенной и необыкновенной 
волн,

$$
\left(\begin{array}{c}
V_{o} \\
V_{e}
\end{array}\right)=R(\beta)\left(\begin{array}{c}
V_{x} \\
V_{y}
\end{array}\right) .
$$

Поскольку обыкновенная и необыкновенная волны ортогональны, матрица Джонса в системе координат, связанной с этими векторами, должна иметь диагональную форму,

$$
D=\left(\begin{array}{cc}
d_{o} & 0 \\
0 & d_{e}
\end{array}\right)
$$

где коэффициенты $d_{i}$ несут информацию об изменении амплитуды и фазы волн при прохождении через образец. Так как показатель преломления у полупроводников велик $\left(n_{o} \sim 3\right)$, расчет матрицы Джонса был проведен с учетом многолучевой интерференции обыкновенной и необыкновенной волн из-за переотражений от передней и задней поверхностей образца. С учетом многократных отражений

$$
d_{i} \sim \frac{1}{1-\alpha_{i}^{2} \exp \left(-2 j k_{i} h\right)} .
$$

Здесь $i=o, e-$ индексы, указывающие на тип волн, $k_{i}$ - волновые векторы, $h$ - толщина образца, $\alpha-$ амплитудный коэффициент отражения, $j$ - мнимая единица,

$$
\alpha_{i}=\frac{n_{i}-1}{n_{i}+1} .
$$

Матрица Джонса в лабораторной системе координат будет выглядеть так:

$$
D=\left(\begin{array}{cc}
d_{o} \cos ^{2} \beta+d_{e} \sin ^{2} \beta & \frac{1}{2}\left(d_{o}-d_{e}\right) \sin 2 \beta \\
\frac{1}{2}\left(d_{0}-d_{e}\right) \sin 2 \beta & d_{e} \cos ^{2} \beta+d_{e} \sin ^{2} \beta
\end{array}\right) .
$$

Вектор Джонса зондирующего излучения после прохождения образца или отражения от него выглядит следующим образом:

$$
\begin{aligned}
& \left(\begin{array}{l}
V_{x} \\
V_{y}
\end{array}\right)= \\
& =E_{o}\left(\begin{array}{l}
\cos \alpha\left(d_{o} \cos ^{2} \beta+d_{e} \sin ^{2} \beta\right)+\sin \alpha \frac{1}{2}\left(d_{o}-d_{e}\right) \sin 2 \beta \\
\sin \alpha\left(d_{o} \cos ^{2} \beta+d_{e} \sin ^{2} \beta\right)+\cos \alpha \frac{1}{2}\left(d_{o}-d_{e}\right) \sin 2 \beta
\end{array}\right) .
\end{aligned}
$$

Появившиеся вторые слагаемые в поляризационных компонентах вектора Джонса (3) могут приводить в графиках к изменениям структуры азимутальных зависимостей ВГ, в частности, к поднятию уровней ряда минимумов и появлению их асимметрии.

Отметим, что этот эффект модификации сигнала ВГ из-за напряжений интенсивно используется в последнее время для вычисления и картирования полей напряжений в различного рода структурах (см., например, [27]).

Многочисленные сравнительные эксперименты показали, что значимое влияние полей напряжений в
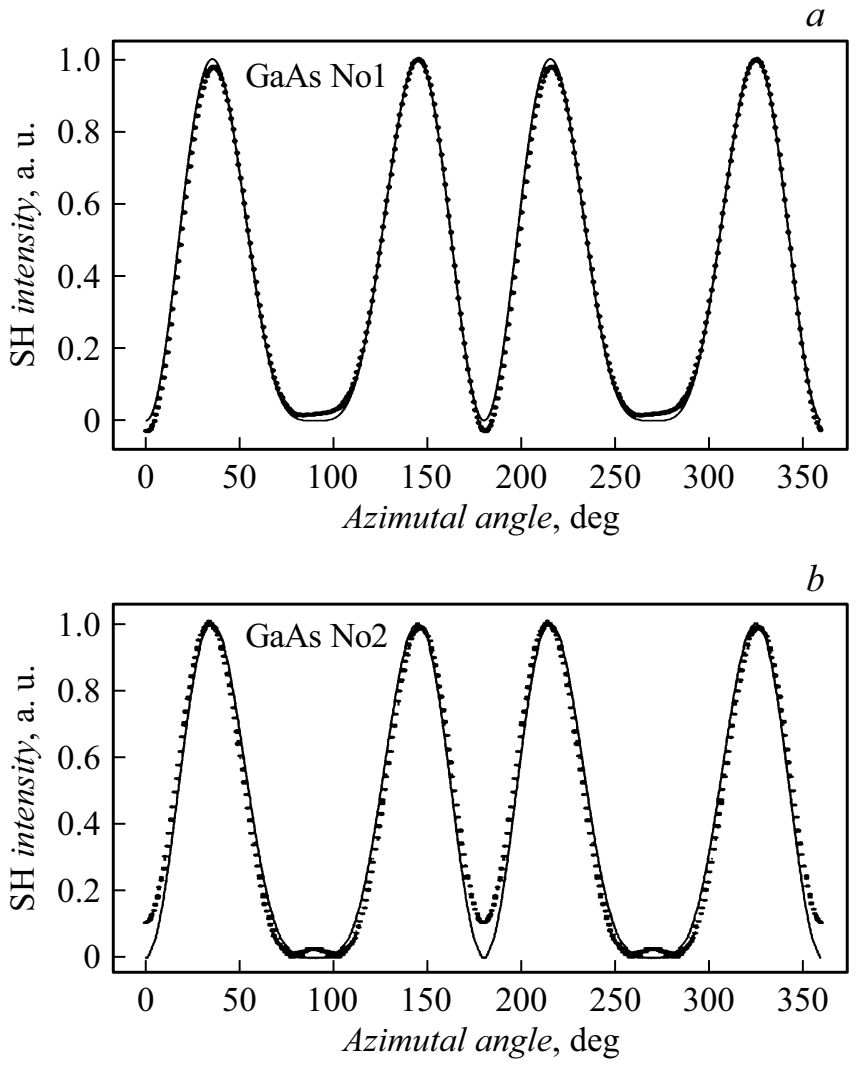

Рис. 4. Результат сравнения экспериментального графика интенсивности сигнала ВГ (топунктирная линия, проведена очистка массива по высоким частотам) с теорией (сплошная линия) для подложек № 1 (a) и № $2(b) \mathrm{GaAs}$. Отклонение от ориентации (013) по углу $\varphi$ составляет менее $1^{\circ}$. Напряжения в подложке № 1 слабые (отсутствуют асимметрия и подъемы у экспериментального графика), в подложке № $2-$ сильные. Средняя мощность излучения на $\lambda=1.064 \mu \mathrm{m}$ равна $0.06 \mathrm{~W}$. Поляризации ВГ и лазерного излучения параллельны.

подложках GaAs и структурах КРТ на положение и относительную величину максимумов сигнала ВГ в азимутальных графиках при сравнении с аналогичными модельными зависимостями для идеального монокристалла проявляется в существенно меньшей степени в силу малости самого эффекта наведенного двулучепреломления.

В качестве наглядных примеров приведем иллюстрации экспериментальных графиков азимутальной зависимости ВГ в сравнении с модельными зависимостями для идеального монокристалла без напряжений.

Сравнение экспериментальных и модельных угловых зависимостей сигнала ВГ от образцов осуществлялось программно с использованием массива модельных угловых зависимостей с вариацией по углу $\varphi$ в $1^{\circ}-$ это и есть погрешность экспериментальных результатов, представленных в настоящей работе.

На рис. 4 приведены экспериментальные и модельные угловые зависимости величины сигнала ВГ от двух подложек GaAs: $a$ - № 1 (без значительных напря- 


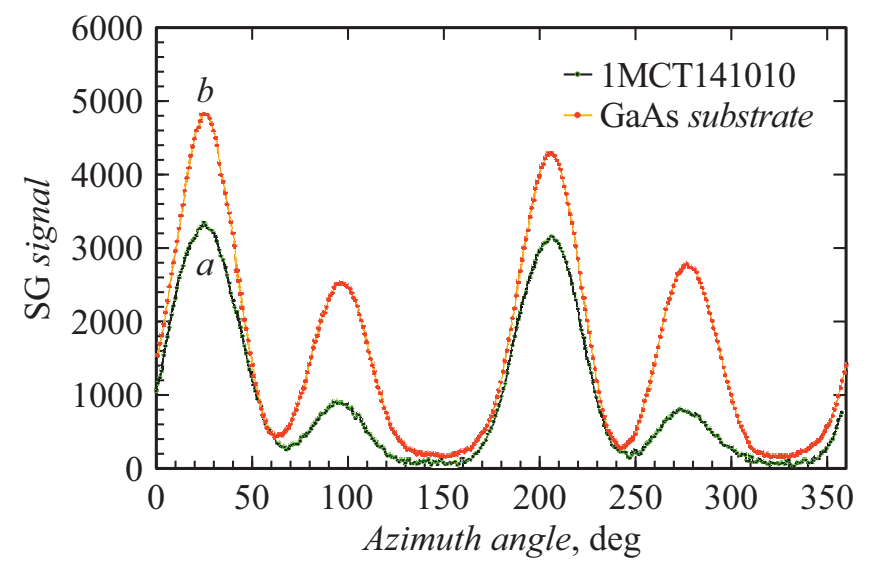

Рис. 5. Экспериментальные графики азимутальной зависимости сигнала ВГ от образца КРТ 141010 (a) и от подложки $\mathrm{GaAs}$ этой гетероструктуры $(b)$. Средняя мощность излучения на $\lambda=1.064 \mu \mathrm{m}$ для $a-$ равна $0.04 \mathrm{~W}$, для $b-0.13 \mathrm{~W}$. Поляризации ВГ и лазерного излучения параллельны. Видны заметные одинаковые напряжения в структуре и подложке (выраженная асимметрия и разные уровни минимумов угловой зависимости у экспериментального графика).

жений) и $b-$ № 2 (явное присутствие напряжений). Здесь экспериментальный график - пунктирная линия (проведена очистка экспериментального массива по высоким частотам), теоретическая угловая зависимость сплошная линия.

Точно таким же образом на большом экспериментальном материале однозначно установлено, что характерное изменение поведения азимутальных зависимостей сигнала ВГ из-за напряжений в подложке проявляется таким же образом и в слоях гетероструктуры, нанесенной на эту подложку. Рис. 5 наглядно демонстрирует этот факт. Здесь для образца КРТ141010 приведены азимутальные зависимости сигнала ВГ от лицевой стороны гетероструктуры КРТ (a) и от тыльной стороны (подложки $\mathrm{GaAs}$ ) этого образца. При сравнении графиков хорошо видно, что напряжения подложки проявляются в сигнале ВГ до самых верхних слоев гетероструктуры КРТ, которая, кроме всего прочего, увеличила по ориентации отклонение от (013) по сравнению с подложкой на $\sim 4^{\circ}$ по углу $\varphi$. Для большей наглядности визуального сравнения минимумов двух экспериментальных графиков средняя мощность возбуждающего излучения при регистрации ВГ от подложки была увеличена в три раза (соответственно сигнал ВГ стал больше приблизительно на порядок).

В рамках настоящей работы мы лишь обращаем внимание на возможности метода ВГ однозначно фиксировать наличие/отсутствие значимых напряжений в подложечном материале и слоях гетероструктур на нем, а также предполагаем возможность расчета азимутальных зависимостей сигнала ВГ в напряженном кристалле методом возмущений, используя модель слабого наведенного двулучепреломления.

\section{4. Воздействие зондирующего излучения повышенной мощности (локальный кратковременный лучевой нагрев) на характеристики кристаллического состояния структур и слоев КРТ}

Так как гетероструктуры КРТ непрозрачны для используемого возбуждающего излучения и для его второй гармоники, нами проведены in situ воздействия мощности используемого лазерного излучения для определения порогового значения мощности, с которого начинают происходить изменения кристаллических характеристик поверхности слоя КРТ выращенной гетероструктуры. Были проведены измерения кристаллического состояния приповерхностной области внешнего варизонного слоя гетероструктуры КРТ (состав 0.45 молярных долей $\mathrm{CdTe})$. Экспериментально было выяснено, что воздействие мощности используемого лазерного излучения начинает оказывать влияние на кристаллические характеристики уже при мощности $\sim 0.2 \mathrm{~W}$ и выше (площадка диаметром $200 \mu \mathrm{m})$. На рис. 5 приведены начальные
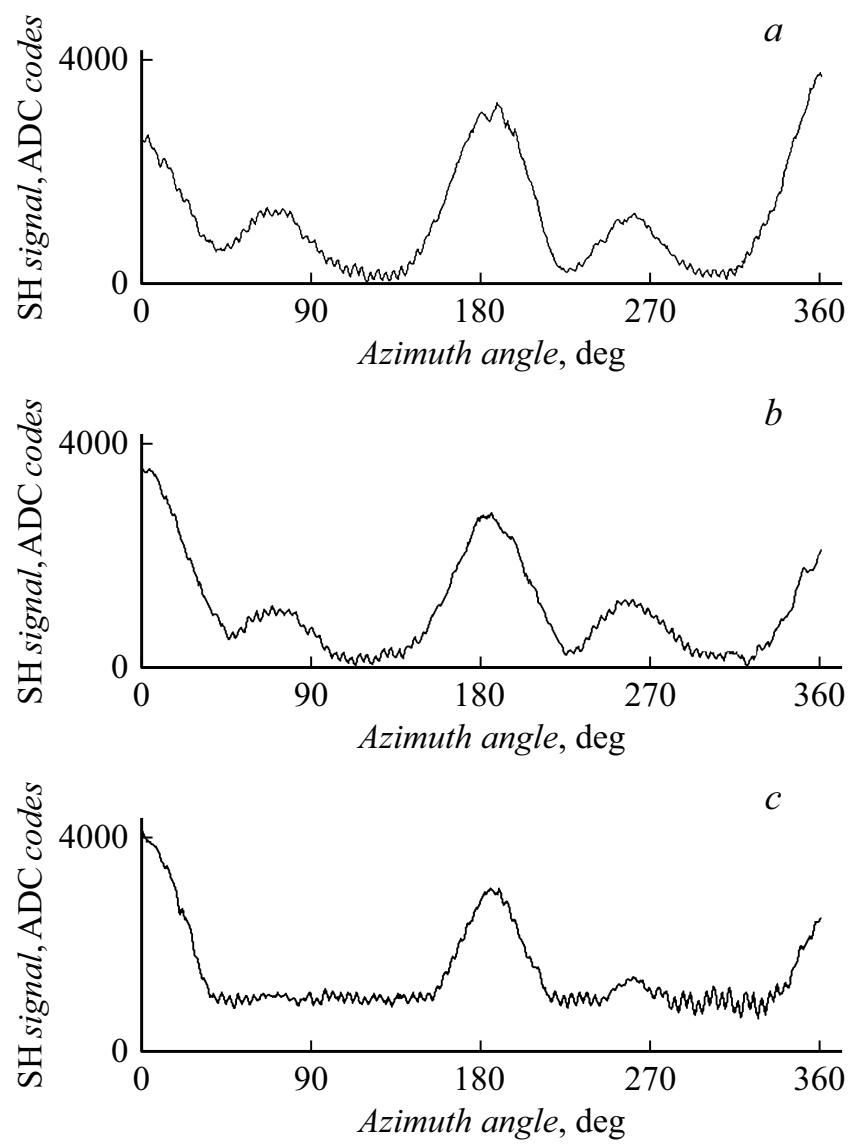

Рис. 6. Азимутальные зависимости сигнала ВГ от одной и той же точки лицевой поверхности образца КРТ 141010 (нормальное падение, $s-s$-геометрия, последовательная непрерывная хронология графиков $a-c$, время записи каждого графика $60 \mathrm{~s}$, интервал между графиками $\sim 7 \mathrm{~s})$. Мощность непрерывно действовавшего на этом временном промежутке возбуждающего излучения $\sim 0.3 \mathrm{~W}$. 
азимутальные зависимости сигнала ВГ, характеризующие кристаллическое состояние поверхности КРТ и тыльной стороны подложки гетероструктуры КРТ141010 при мощностях зондирующего излучения 0.04 и $0.13 \mathrm{~W}$ соответственно.

При больших мощностях наблюдались изменения кристаллического состояния приповерхностного слоя КРТ. На рис. 6 приведены данные при воздействии возбуждающим излучением мощностью $0.3 \mathrm{~W}$ при записи азимутальной зависимости сигнала ВГ в течение $60 \mathrm{~s}$. Для доказательства того, что тонкая структура азимутальных зависимостей на рис. 6 не связана с шумами АЦП (士16 единиц кода АЦП), амплитуда регистрируемого сигнала ВГ приведена в кодах АЦП. Из приведенных результатов следует, что при воздействии в течение $194 \mathrm{~s}$ происходит уменьшение амплитуды сигнала ВГ в максимумах и возрастание шумов в минимумах (рис. $6, c$ ). Это значит, что в течение этого времени происходит локальный кратковременный нагрев возбуждающим излучением до физического изменения кристаллического состояния, которое не восстанавливается полностью к первоначальному состоянию. Действительно, в процессе нагрева локальная приповерхностная зона от начального состояния (рис. 6,a) постепенно теряла свойства симметрии своего класса при сохранении общей нецентросимметричности гетероструктуры (рис. $6, b, c)$, появлялись разупорядоченные микроучастки гетероструктуры КРТ (см. разд. 3.2), дававшие диффузную составляющую в сигнал ВГ. Таким образом, наблюдается локальный лазерный отжиг, при котором кристаллическое состояние материала может изменять свое состояние вплоть до появления аморфной фазы, как показано в [1]. Вопросы о модификации свойств слоев гетероструктуры во время воздействия и о конечных кристаллических характеристиках облученной зоны после лучевого воздействия являются предметом дальнейших исследований.

\section{Заключение}

Представлены результаты по созданию и применению высокочувствительной экспрессной нелинейнооптической диагностики на основе метода генерации второй гармоники и измерений азимутальных угловых зависимостей поляризационных компонент сигнала отраженного от образца ВГ зондирующего лазерного излучения. Осуществлено повышение чувствительности нелинейно оптической диагностики с целью получения более информативной характеризации кристаллов типа сфалерита. Получены новые экспериментальные результаты кристаллического качества ГЭС КРТ МЛЭ и подложечного материала.

Проведенные исследования гетероструктур твердого раствора теллурида кадмия и ртути, выращенных на подложках из $\mathrm{GaAs}$ методом молекулярно-лучевой эпитаксии, с помощью высокочувствительного стенда НОД подтвердили влияние напряжений на выраженную асимметрию минимумов угловой зависимости экспериментальных графиков сигнала ВГ в подложках $\mathrm{GaAs}$ и слоях КРТ. Зарегистрировано одинаковое проявление на графиках сигнала ВГ напряжений в подложечном материале и в слоях выращенной гетероструктуры КРТ.

По регистрации сигнала отраженной ВГ от слоев $\mathrm{HgCdTe}$ и подложки GaAs подтверждено, что величина нелинейной восприимчивости $\chi_{x y z}(\omega)$ КРТ зависит от состава слоев КРТ и превосходит, по крайней мере, на порядок аналогичную величину для CdTe и GaAs на длине волны лазерного излучения $\sim 1 \mu \mathrm{m}$. Величина нелинейной восприимчивости для слоев КРТ состава 0.22 молярных долей СdТе превосходит аналогичное значение для слоев КРТ состава 0.45 молярных долей $\mathrm{CdTe}$.

Выявлен эффект повышения шума в минимумах азимутальной зависимости в слоях КРТ в некоторых гетероструктурах, значительно превышающий шум измерительного тракта. Такое поведение нами связывается с присутствием разориентированных микроучастков в слоях гетероструктуры КРТ, что наблюдается впервые.

Определено пороговое значение мощности возбуждающего излучения менее $0.2 \mathrm{~W}$, при котором не наблюдается изменений кристаллического состояния в слоях КРТ, что подтверждает корректность измерений состояния кристаллической структуры после выращивания.

При более высоких мощностях $\sim 0.2-0.3 \mathrm{~W}$ наблюдается локальный кратковременный лучевой нагрев, приводящий к изменениям кристаллической структуры слоев в гетероструктуре КРТ. Явление наблюдалось in situ по одновременным измерениям азимутальных зависимостей сигнала ВГ. Таким способом можно контролируемо изменять состояние кристаллической структуры и проводить локальную модификацию свойств материала.

Показано, что метод характеризации кристаллического состояния структур и слоев КРТ по второй гармонике позволяет осуществлять экспрессное сопровождение технологии создания полупроводниковых гетероструктур КРТ для достижения ими требуемого качества при последующих применениях.

\section{Благодарности}

Работа выполнена при частичной поддержке Российского фонда фундаментальных исследований (проект № 18-29-20053), Volkswagen Stiftung Program (№ 97738) и в рамках государственного задания Минобрнауки России в части проекта АААА-А20-120102190007-5.

\section{Конфликт интересов}

Авторы заявляют, что у них нет конфликта интересов.

\section{Список литературы}

[1] С.А. Ахманов, В.И. Емельянов, Н.И. Коротеев, В.В. Семиногов. УФН, 147 (12), 675 (1985).

[2] T.F. Heinz. Second-Order Nonlinear Optical Effects at Surfaces and Interfaces. In: Nonlinear Surface Electromagnetic Phenomena. Eds H. Ponath, G. Stegeman. (North Holland Pub., Амстердам, 1991) 
[3] T. Kimura, Ch. Yamada, J. Crystal Growth, 150, 92 (1995).

[4] К.А. Брехов, К.А. Гришунин, Д.В. Афанасьев, С.В. Семин, Н.Э. Шерстюк, Е.Д. Мишина, А.В. Кимель. ФТТ, 60 (1), С 33 (2018).

[5] В.В. Баланюк, В.Ф. Краснов, С.Л. Мушер, В.И. Проц, В.Э. Рябченко, С.А. Стоянов, С.Г. Струц, М.Ф. Ступак, В.С. Сыскин. Квантовая электроника, 22 (2), 196 (1995).

[6] Г.М. Борисов, В.Г. Гольдорт, А.А. Ковалев, С.А. Кочубей, Д.В. Ледовских, В.В. Преображенский, М.А. Путято, Н.Н. Рубцова, Б.Р. Семягин. Вестн. Новосиб. гос. ун-та. Серия: Физика, 9 (4), 5 (2014).

[7] Г.М. Борисов, В.Г. Гольдорт, К.С. Журавлев, А.А. Ковалев, С.А. Кочубей, Д.В. Ледовских, Т.В. Малин, Н.Н. Рубцова. Сибирский физический журнал, $13(2), 64$ (2018). DOI: 10.25205/2541-9447-2018-13-2-64-69

[8] С.Б. Бодров, А.И. Корытин, Ю.А. Сергеев, А.Н. Степанов. Квантовая электроника, 50 (5), 496 (2020). [S.B. Bodrov, A.I. Korytin, Yu.A. Sergeev, A.N. Stepanov. Quant. Electron., 50 (5), 496 (2020).] DOI: http://dx.doi.org/10.1070/QEL17185

[9] И.Д. Бурлаков, А.В. Демин, Г.Г. Левин, Н.А. Пискунов, С.В. Заботнов, А.С. Кашуба. Измерительная техника, 6, 15 (2010).

[10] Е.В. Пермикина, А.С. Кашуба. Успехи прикладной физики, 4 (5), 493 (2016).

[11] М.Ф. Ступак, Н.Н. Михайлов, С.А. Дворецкий, М.В. Якушев, Д.Г. Икусов, С.Н. Макаров, А.Г. Елесин, А.Г. Верхогляд. ФТТ, 62 (2), 214 (2020). DOI: 10.21883/FTT.2020.02.48870.601 [M.F. Stupak, N.N. Mikhailov, S.A. Dvoretskii, M.V. Yakushev, D.G. Ikusov, S.N. Makarov, A.G. Elesin, A.G. Verkhoglyad. Physics of the Solid State, 62 (2), 252 (2020). DOI: $10.1134 / \mathrm{S} 1063783420020201]$

[12] V.V. Pavlov, A.M. Kalashnikova, R.V. Pisarev, I. Sanger, D.R. Yakovlev, M. Bayer. J. Opt. Soc. Am. B, 22, 168 (2005).

[13] M. Fiebig, V.V. Pavlov, R.V. Pisarev. J. Opt. Soc. Am. B, 22, 96 (2005).

[14] С.Л. Мушер, М.Ф. Ступак, В.С. Сыскин. Квантовая электроника, 23 (8), 762 (1996).

[15] A. Rogalski. Rep. Prog. Phys., 68, 2267 (2005).

[16] Ю.Г. Сидоров, С.А. Дворецкий, В.С. Варавин, Н.Н. Михайлов, М.В. Якушев, И.В. Сабинина. ФТП, 35 (9), 1092 (2001).

[17] Ю.Г. Сидоров, М.В. Якушев, А.В. Колесников. Автометрия, 50 (3), 25 (2014).

[18] Ю.Г. Сидоров, М.В. Якушев, В.С, Варавин, А.В. Колесников, Е.М. Труханов, И.В. Сабинина, И.Д. Лошкарев. ФТТ, 57 (11), 2095 (2015).

[19] D. Chandra, H.D. Shih, F. Aqariden, R. Dat, S. Gutzler, M.J. Bevan, T. Orent. J. Electron. Mater., 27 (6), 640 (1998).

[20] L. He, Y. Wu, L. Chen, S.L. Wang, M.F. Yu, Y.M. Qiao, J.R. Yang, Y.J. Li, R.L. Ding, Q.Y. Zhang. J. Cryst. Growth, 227-228, 677 (2001).

[21] J.D. Benson, L.O. Bubulac, P.J. Smith, R.N. Jacobs, J.K. Marcunas, M. Jaime-Vasques, L.A. Almeida, A.J. Stoltz, P.S. Wijewarnasuriya, G. Brill, Y. Chen, U. Lee, M.F. Vilela, J. Peterson, S.M. Johnson, D.D. Lofgreen, D. Rhiger, E.A. Patten, P.M. Goetz. J. Electron. Mater., 39(7), 1080 (2010).

[22] В.И. Гавриленко, А.М. Грехов, Д.В. Корбутяк, В.Г. Литовченко. Оптические свойства полупроводников. Справочник (Наукова думка, Киев, 1987)

[23] Handbook of Optical Constants of Solids. Ed. by E.D. Palik. (Elsevier Science, USA, 1998)
[24] Optical Constants of Crystalline and Amorphous Semiconductors. Sadao Adachi. (Springer Science+ Business Media, NY., 1999)

[25] E.D. Mishina, T.V. Misuryaev, N.E. Sherstyuk, V.V. Lemanov, A.L. Morozov, A.S. Sigov, Th. Rasing. Phys. Rev. Lett., 85, 3664 (2000).

[26] Е.Д. Мишина, А.И. Морозов, А.С. Сигов, Н.Е. Шерстюк, O.А. Акципетров, В.В. Леманов, Th. Rasing. ЖЭТФ, 121, (3), 644 (2002).

[27] L. Mennel, M.M. Furchi, S. Wachter, M. Paur, D.K. Polyushkin, Th. Mueller. Nat. Commun., 9, 516 (2018). DOI: 10.1038/s41467-018-02830-y

[28] B.R. Carvalho, Y. Wang, K. Fujisawa, T. Zhang, E. Kahn, I. Bilgin, P.M. Ajayan, A.M. de Paula, M.A. Pimenta, S. Kar, V.H. Crespi, M. Terrones, L.M. Malard. Nano Lett., 20 (1), 284 (2020). DOI: 10.1021/acs.nanolett.9b03795 